Ann. Biol. anim. Bioch. Biophys., I973, 13 (I), 75-92.

\title{
TRANSFORMATION ET DEVENIR DES GELLULES ADIPEUSES AU COURS DE L'AMAIGRISSEMENT. ÉTUDE ULTRASTRUCTURALE
}

\author{
F. DESNOYERS, N. VODOVAR et G. DURAND \\ avec la collaboration technique de S. DeLPAL \\ Station de Recherches de Nutrition, \\ Centre national de Recherches zootechniques, I. N. R. A., \\ 78350 Jouy en Josas
}

\begin{abstract}
RÉSUMÉ
Chez des rats de quinze semaines pesant environ $400 \mathrm{~g}$, les transformations ultrastructurales des cellules adipeuses et leur devenir ont été étudiés au cours de la déplétion lipidique provoquée par un régime de restrictions prolongées en éléments énergétiques et dans des conditions aussi physiologiques que possible. Il a été montré que les adipocytes, parvenus au stade terminal de leur évolution, au moment de la mise au régime restrictif des animaux, sont dégradés et disparaissent généralement avant que ces animaux aient perdu $20 \mathrm{p}$. roo de leur poids initial.

Le reste des cellules adipeuses des dépôts, représentant environ $90 \mathrm{p}$. Ioo de la totalité des cellules ayant des inclusions lipidiques, subissent des transformations structurales et ultrastructurales leur permettant de se régénérer tout en perdant leurs lipides d'accumulation.

Il a été constaté que les modifications structurales et ultrastructurales de ces cellules adipeuses au cours du départ des lipides sont d'autant plus spectaculaires que leurs inclusions lipidiques sont plus importantes au moment de la mise au régime des animaux.

Les différentes étapes de ces transformations, jusqu'à la déplétion totale des lipides qui est généralement achevée lorsque les animaux ont perdu $50 \mathrm{p}$. roo de leur poids initial, ont été décrites et discutées.
\end{abstract}

\section{INTRODUCTION}

Chez les rats à croissance normale, l'ultrastructure des cellules adipeuses à graisses blanches, dépourvues de lipides d'accumulation par amaigrissement provoqué, a fait l'objet de plusieurs travaux au cours des dix dernières années. Les investigations, suivant les chercheurs, ont été faites soit sur des animaux complète- 
ment privés de nourriture jusqu'à la perte de leurs lipides d'accumulation ou sur des animaux diabétiques (SHELDON, Hol, ENBERG, WINEGrad, I962), soit sur des animaux soumis à des restrictions caloriques (NAPOLITANO, GAGNE, I963) ou encore sur des animaux privés de nourriture pendant six à dix jours et soumis par la suite à des restrictions caloriques (SIMON, WILLIAMSON, I967). Les renseignements obtenus par ces travaux, effectués dans des conditions expérimentales différentes, concernent uniquement les cellules adipeuses au stade complet de leur déplétion lipidique tandis que les transformations dont elles sont l'objet pendant la mobilisation des lipides n'ont pas été étudiées jusqu'à présent.

Du fait que les dépôts adipeux normaux sont à tout moment constitués par des cellules adipeuses aux différents degrés de leur évolution, c'est-à-dire par des adipocytes parvenus au stade terminal de leur maturation, par des cellules contenant peu d'inclusions lipidiques et par des cellules à des stades intermédiaires (VoDovar, DESNOYERS, FlANZY, I972), on peut penser que les modes de transformation et le devenir de l'ensemble des populations de cellules adipeuses ne sont pas obligatoirement les mêmes au cours de 1'amaigrissement.

Les présentes observations sur les dépôts mésentériques ont été faites dans des conditions aussi physiologiques que possible et en tenant compte des besoins protéiques pour que la lipolyse ne soit pas inhibée. Elles ont pour but d'étudier les cellules adipeuses aux différents moments de leur déplétion lipidique, de préciser la possibilité de leur régénération et d’indiquer le mode de dégradation éventuel de certaines d'entre elles.

La nomenclature employée en ce qui concerne les cellules aux différents stades de l'accumulation a été définie précédemment (Vodovar, DESNOYERS, Flanzy, I972), sauf pour les cellules ayant perdu leurs lipides d'accumulation. Nous pensons que ces dernières pourront être considérées comme postadipocytes par analogie avec les préadipocytes, sans inclusions lipidiques.

\section{MATÉRIEL ETT MÉTHODES}

Des lots de rats Wistar âgés de quinze semaines, d'un poids d'environ 4 oo g, ont été soumis à un régime de restrictions prolongées en éléments énergétiques (lipides et glucides), tout en recevant autant de protéines, de minéraux et de vitamines qu'en consommeraient des animaux de même âge, de même poids et nourris à volonté. L'amaigrissement, dans ces conditions, est en moyenne de $3 \mathrm{~g}$ par 24 heures. L'abattage des animaux a été réparti entre le sixième et le soixantequinzième jour de régime, ce qui correspond à une perte comprise entre 5 et 55 p. I oo de leur poids initial. Pour les pertes de poids inférieures à 5 p. Ioo, il n'a pas été observé de transformations ultrastructurales des cellules adipeuses, tandis que les cellules adipeuses des dépôts mésentériques des animaux ayant perđu $5^{\circ}$ p. Ioo de leur poids initial sont pratiquement dépourvues de leurs lipides d'accumulation dans tous les cas observés. Des observations préliminaires effectuées chez de jeunes rats de sept semaines, pesant en moyenne $240 \mathrm{~g}$, nous ont montré que l'évolution des cellules adipeuses au cours de la déplétion lipidique n'est pas toujours identique à celle des cellules de rats adultes faisant l'objet de cette étude.

La fixation avec le tétroxyde d'osmium à 2 p. Ioo dans le tampon phosphaté à o, I M (MiLLONIG, I96I) a été pratiquée in vivo pendant 5 à Io minutes puis après extirpation des tissus pendant 12 heures, soit à $4^{\circ} \mathrm{C}$, soit à la température du laboratoire. L'inclusion dans l'Épon et la confection des coupes avec l'Ultratome LKB III ont été faites suivant les techniques déjà décrites (Vodovar, Desnoyers, François, I97I). Les coupes ont été observées avec l'Elmiscop Siemens IA. La détermination de la cellularité, comme dans le travail précédent (Vodovar, Desnoyers, Flanzy, 1972), a été faite par recoupement de différentes techniques afin de parvenir à une évaluation aussi satisfaisante que possible du nombre réel de cellules. 


\section{OBSERVATIONS}

\section{I. - Tissu adipeux d'animaux témoins nourris normalement}

Sur les coupes des dépôts adipeux de rats âgés de quinze semaines, pesant environ $400 \mathrm{~g}$ et recevant un régime équilibré, on observe de très nombreux adipocytes, c'est-à-dire des cellules adipeuses dont les lipides d'accumulation ont déjà fusionné en une seule masse, un certain nombre de cellules avec des inclusions lipidiques d'importance variable, de rares cellules présumées être les futurs adipocytes, des mastocytes, des éléments du tissu conjonctif, du tissu endothélial et du tissu nerveux. Ces différents constituants des tissus adipeux, que nous comparerons avec ceux des rats amaigris, ont été déjà étudiés sur plusieurs espèces aussi bien en ce qui concerne leur répartition topographique dans le dépôt que leur aspect ultrastructural (Wassermani, Mc Donald, I960 $a$; Napolitano, I963; Vodovar, Desnoyers, Françors, I97I ; Vodovar, Desnoyers, Flanzy, I972) ; nous ne reviendrons pas ici sur leur description.

\section{II. - Tissus adipeux des animaux soumis à l'amaigrissement}

Dans les conditions de nos observations, l'importance quantitative des lipides mobilisés est liée au degré d'amaigrissement des animaux soumis au régime lipidoprive. Les cellules adipeuses dont les transformations ultrastructurales sont consécutives au départ des lipides ont un aspect différent selon les étapes de 1'amaigrissement. Ces étapes, grossièrement délimitées, correspondent à une perte de poids inférieure à $20 \mathrm{p}$. IOO, puis à une perte de poids comprise entre 20 et $50 \mathrm{p}$. IOO, enfin à une perte supérieure à $50 \mathrm{p}$. Ioo du poids enregistré au début du régime.

\section{a) Cellules adipeuses des animaux ayant perdu 5 à 20 p. 100 de leur poids.}

La comparaison entre les coupes de tissu adipeux prélevé sur les animaux ayant perdu, en fonction de la durée du régime, de 5 à $20 \mathrm{p}$. Ioo de leur poids initial et les coupes de tissu adipeux prélevé à des niveaux correspondants sur les animaux témoins, montre que le départ des lipides a engendré des transformations structurales et ultrastructurales des cellules adipeuses. Nous ne savons pas actuellement par quel mécanisme et dans quel rapport la mobilisation des lipides se répartit dans l'ensemble des populations de cellules adipeuses, compte tenu de leur emplacement et de leur degré d'évolution. Néanmoins, on constate que les modifications ultrastructurales mises en évidence pendant la première phase de l'amaigrissement sont d'autant plus apparentes que la maturation des cellules adipeuses est plus avancée au moment de la mise au régime des animaux. Ainsi, suivant les modes et l'importance des transformations, l'ensemble des cellules adipeuses peut être réparti en trois groupes qui sont respectivement : les adipocytes parvenus au stade terminal de leur maturation, les adipocytes au cours de leur évolution et les préadipocytes à multiples inclusions lipidiques. 
I. Déplétion lipidique des adipocytes parvenus au stade de leur maturation (fig. A).

Numériquement peu importantes (5 à Io p. IOo), les cellules de ce groupe, relativement homogènes au début de l'amaigrissement, se caractérisent par une masse lipidique importante de diamètre généralement compris entre IIo et I30 $\mu$ et par un anneau périphérique réduit (fig. I) dont l'épaisseur est souvent inférieure à I $\mu$ excepté au niveau du noyau. Les organites cellulaires disséminés dans l'anneau périphérique sont peu nombreux et souvent en état de dégradation ; la matrice fondamentale du cytoplasme ne se distingue pas dans la majorité des cas. Après environ cinq jours de régime, on observe la dégradation rapide des éléments cellulaires encore présents sur le pourtour de la masse lipidique des adipocytes. Cette dégradation, sans avoir toujours le même aspect, se caractérise en premier lieu par la détérioration progressive de la lame basale et de la membrane plasmique. La présence dans ce qui reste de l'anneau périphérique de microvésicules entremêlées de fibres conjonctives de diamètre et d'orientation variables est fréquente (fig. 2). De la disparition assez rapide des éléments périphériques des adipocytes, il résulte que la masse lipidique, dans certains cas, reste bordée par une enveloppe très fine et sans structure apparente (fig. 3). Dans d'autres cas, les fibres conjonctives restent seules en contact avec les lipides (fig. 4) ou encore la masse lipidique est pratiquement dépourvue de toute substance organisée sur son pourtour (fig. 5). On note également que le volume des lipides diminue souvent plus rapidement que la surface cellulaire, ce qui entraine une séparation entre la masse lipidique et l'anneau périphérique qui, alors, disparaît rapidement.

Les transformations aboutissant à la dégradation des éléments cellulaires de l'anneau périphérique de ces adipocytes laissent à penser que les cellules adipeuses, parvenues au stade terminal de leur maturation, ne se régénèrent pas quand elles sont soumises à un régime restrictif. Le départ des lipides d'accumulation a pour conséquence leur dégradation et leur disparition rapides. Le nombre d'adipocytes dégradés et disparus nous paraît relativement peu élevé ; il peut être grossièrement évalué de 5 à Io p. Ioo du nombre total des cellules adipeuses.

2. Déplétion lipidique des adipocytes aux différents stades de leur évolution (fig. B).

Ce groupe de cellules adipeuses, très polymorphes, est quantitativement le plus important. Leur nombre, chez les rats de quinze semaines, est estimé, au début de 1'amaigrissement, de 60 à 75 p. Ioo du nombre total des cellules ayant des inclusions lipidiques. L'hétérogénéité des cellules doit être attribuée à un degré variable d'évolution lié à la quantité de lipides dont dépend le volume de la cellule et à l'importance de 1'anneau périphérique. Dans les conditions de 1'expérience, les transformations ultrastructurales de ces cellules deviennent surtout importantes à partir du moment où les animaux ont perdu environ 5 p. Ioo de leur poids. Les premières transforma-

FIG. A. - Représentation schématique de trois stades de l'évolution d'un adipocyte qui était parvenu au stade terminal de sa maturation au moment de la mise au régime lipidoprive des animaux. Dans ce cas, la perte des lipides d'accumulation provoquera la dégradation puis la disparition de la cellule adipeuse. Stade $a$. - Une importante masse lipidique L est entourée par un anneau périphérique AP réduit. Noyau : $\mathrm{N}$; les mitochondries, $M$, sont en nombre très réduit.

Stade $b$. - L'anneau périphérique AP, ne s'adaptant pas au départ des lipides se décolle de la masse lipidique $L$ avec apparition d'un espace vide $E V$, est en état de dégradation.

Stade $c$. - La déplétion lipidique augmentant, l'espace vide EV se trouve fortement accentué et l'anneau périphérique AP continue sa dégradation jusqu'à complète disparition. Lipides : $L$. 

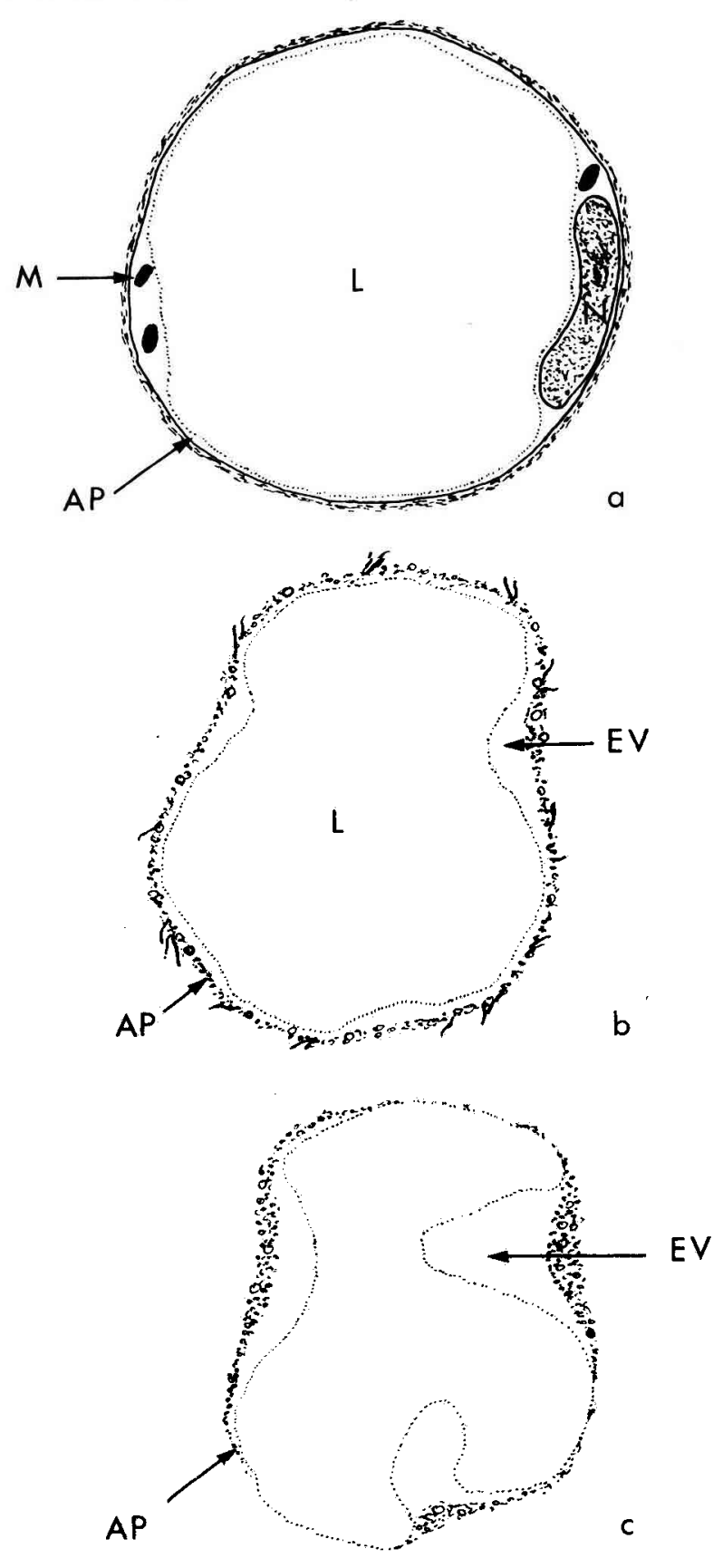

FIG. A 
tions paraissent être principalement liées à une réduction du volume cellulaire par mobilisation des lipides. Pendant la régression du volume des adipocytes, ces cellules, qui auparavant sont fréquemment en contact entre elles ou avec les cellules endothéliales, ont tendance à s'individualiser (fig. 6).

Contrairement aux adipocytes parvenus au stade terminal de leur maturation, ces cellules ne se dégradent pas (sauf exception), leur lame basale et leur membrane plasmique sont généralement bien apparentes (fig. 7). Les fibres conjonctives qui, dans le dépôt normal, relient souvent les adipocytes en s'entremêlant avec la lame basale et parfois avec l'anneau périphérique, s'éloignent dans la plupart des cas de la surface des cellules et semblent diminuer en nombre et en diamètre.

La forme de ces adipocytes change d'autant plus brutalement que le volume des lipides intracellulaires est plus important et que le diamètre de l'anneau périphérique est plus réduit. Des transformations spectaculaires sont observées dans de nombreux cas au cours de la déplétion lipidique (fig. 7) ; cependant toutes les cellules adipeuses, sauf exception, conservent leur intégrité et survivent au départ des lipides. Les mécanismes de ces transformations sont complexes; la diminution de volume des cellules due au départ des lipides d'accumulation se traduit d'abord par des invaginations, des aplatissements et des plissements de la surface cellulaire (fig. 8).

Les lipides, qui, dans un premier temps, sont présents entre ces plissements, disparaissent progressivement en faisant apparaître des projections cytoplasmiques dont le nombre, l'importance et la forme sont variables (fig. 9).

Dans un certain nombre de cas, les projections cytoplasmiques ne parvenant pas à s'intégrer au reste de la cellule sont détachées et dégradées sans que l'adipocyte disparaisse. On observe, chez tous les adipocytes, simultanément avec ces transformations, l'apparition d'innombrables microinvaginations de la membrane plasmique et de microvésicules pinocytotiques à l'intérieur de l'anneau périphérique (fig. $7,8,9$ ). A ce stade d'amaigrissement, la matrice fondamentale du cytoplasme est généralement répartie de façon très hétérogène et différemment contrastée selon les régions de la cellule. Les mitochondries, en nombre variable suivant le degré d'évolution des adipocytes avant le régime, sont dispersées, exception faite dans de rares projections cytoplasmiques où elles sont déjà regroupées en raison du départ des lipides et de la diminution importante du volume cellulaire (fig. 7). L'appareil de Golgi est presque toujours absent ; le RER et le REL sont, suivant l'importance de l'anneau périphérique, en quantité variable ou absents. Le noyau est en général dans une position périphérique; son aspect et sa forme ne paraissent pas changer par rapport à ce qu'ils étaient au début de l'amaigrissement. I a masse lipidique homogène est devenue plus osmiophile excepté sur son pourtour où, en revanche, elle l'est moins (fig. 7).

FIG. B. - Schéma des différents stades de l'évolution d'un adipocyte pour lequel la déplétion lipidique intervient au cours de sa maturation. Dans ce cas, la cellule subira des transformations spectaculaires (projections cytoplasmiques) pour aboutir à une cellule sans lipides.

Stade $a$. - La cellule adipeuse dont l'anneau périphérique AP, relativement bien conservé, entoure une masse lipidique $\mathrm{L}$ importante, est bordée par une lame basale B d'aspect classique. Noyau : $\mathrm{N}$; Mitochondrie : $\mathbf{M}$.

Au cours des stades $b, c$ et $d$, la masse lipidique $\mathrm{L}$ diminue progressivement de volume (se scinde en plusieurs inclusions) ce qui entraîne une réduction de la taille de la cellule. Au cours de ces transformations, on note la formation de projections cytoplasmiques PC et le plissement de la lame basale B. Le stade $e .-$ représente la cellule adipeuse dépourvue de tout lipide. 


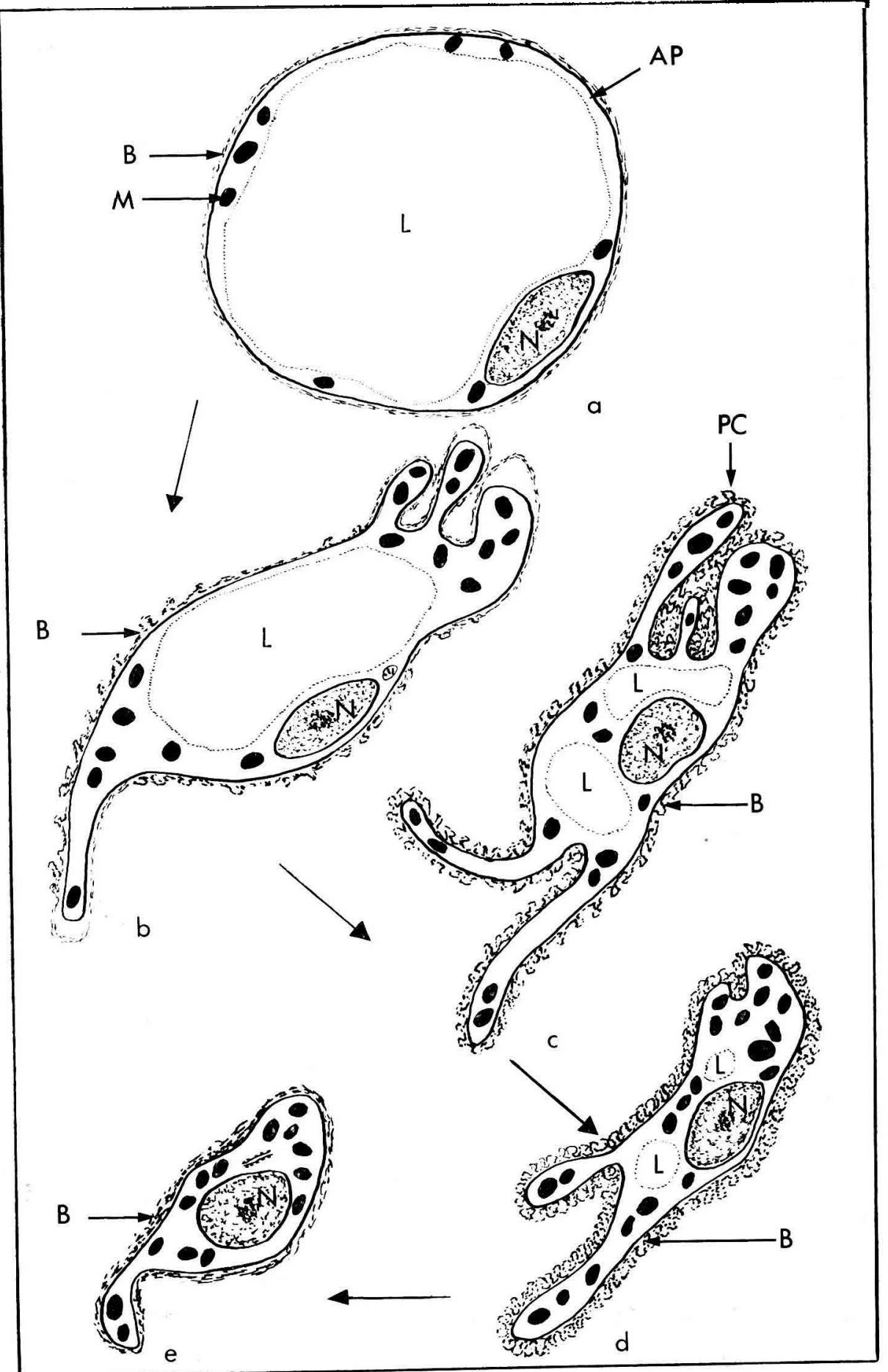

FIG. B 


\section{Déplétion lipidique des cellules adipeuses avec de multiples inclusions lipi-} diques (fig. C).

Comme dans le groupe précédent, les cellules à multiples inclusions lipidiques sont polymorphes; leur importance numérique peut, dans le cas de nos investigations, être approximativement évaluée entre 20 et $30 \mathrm{p}$. Ioo de l'ensemble des cellules adipeuses au début de l'amaigrissement. L'importance des transformations structurales et ultrastructurales de ces cellules nous paraît surtout dépendre du nombre des inclusions lipidiques et de leur volume; par comparaison avec les deux groupes étudiés ci-dessus, les transformations de ces cellules ne sont que peu importantes. Néanmoins, on observe, comme pour les cellules adipeuses du groupe précédent (fig. Io) l'individualisation ainsi que l'apparition de microinvaginations dans la membrane plasmique surtout importantes à proximité des grosses inclusions lipidiques. A l'intérieur des cellules, l'aspect des organites cellulaires et de la matrice fondamentale du cytoplasme reste apparemment sans changement. Le nombre et la taille des inclusions lipidiques sont peu modifiés au cours de cette première étape de l'amaigrissement ; cela conduit à penser, sous condition de confirmation, que la mobilisation des lipides demeure faible pendant cette étape dans ce groupe de cellules.

Au terme de la première étape de l'amaigrissement et dans nos conditions d'expériences, on peut conclure que les cellules adipeuses ont perdu une partie de leurs lipides d'accumulation et que la quantité d'adipocytes disparus est relativement faible, tandis que l'appauvrissement du dépôt en d'autres composants, non mentionnés dans ce travail, et en particulier en éléments du tissu conjonctif nous paraît bien plus important.

\section{b) Cellules adipeuses des animaux ayant perdu de 20 à 50 p. 100 de leur poids.}

I a seconde étape de l'amaigrissement amènera les animaux à des pertes de 50 p. Ioo de leur poids initial et à la disparition quasi totale des lipides d'accumulation, aussi bien des adipocytes que des préadipocytes. Au début de cette seconde étape, ces cellules tout en étant très polymorphes, sont parvenues par de multiples transformations à un stade à partir duquel la plupart des éléments cellulaires de même ordre évoluent d'une façon semblable et avec moins de perturbations apparentes que pendant la première étape du régime. On note également que la disparité de taille des cellules adipeuses est devenue, dans une certaine mesure, moins importante du fait de la disparition des adipocytes les plus volumineux et, sans doute, parce que la mobilisation des lipides d'accumulation est plus rapide à partir des cellules de grande surface. En raison de l'évolution relativement homogène des cellules adipeuses durant cette seconde étape, il nous a paru préférable d'étudier successivement, pour l'ensemble de la population cellulaire, les transformations des différents éléments des cellules adipeuses, ce qui n'a pas été possible au cours de la premì̀re étape de 1'amaigrissement.

FIG. C. Représentation schématique des différentes étapes de l'évolution d'un préadipocyte avec plusieurs inclusions lipidiques $L$ au cours de la perte des lipides d'accumulation. Chez les préadipocytes, les transformations dues à la déplétion lipidique se font harmonieusement pour aboutir à une cellule sans lipides comparable à celle du stade e de la figure $B$.

Noyau : $\mathbf{N}$; Mitochondrie : $\mathbf{M}$; Réticulum endoplasmique rugueux : RER 


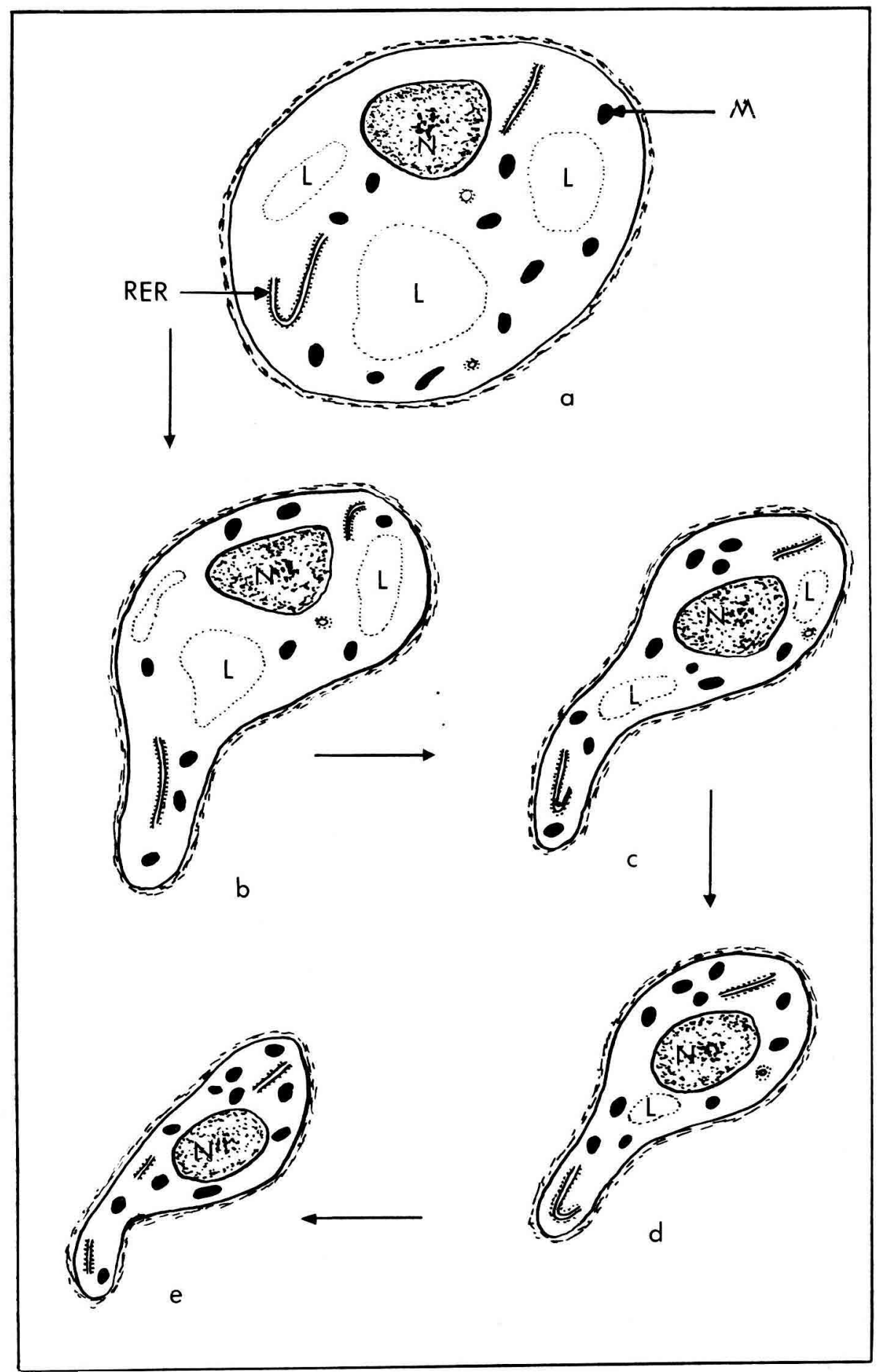

FIG. C 
I. Aspect des lipides d'accumulation au cours de la seconde étape de l'amaigrissement.

Les transformations structurales et ultrastructurales des cellules adipeuses étant la conséquence directe ou indirecte de la mobilisation des lipides, il nous a paru rationnel d'étudier en premier lieu l'évolution de la masse lipidique.

Aux premiers temps de la seconde étape de l'amaigrissement, pour deux tiers environ des cellules adipeuses, les lipides occupent entre 50 et $85 \mathrm{p}$. Ioo du volume cellulaire, le reste de la population en ayant moins de $50 \mathrm{p}$ roo et souvent moins de Io p. Ioo. Vers la fin de la seconde étape de l'amaigrissement, on constate que le nombre des préadipocytes a augmenté par rapport à celui de l'étape précédente, alors que celui des adipocytes jusqu'alors majoritaires, décroît progressivement. Les observations, à ce stade, mettent en évidence des transformations cellulaires qui aboutissent, dans la majorité des cas, à la séparation de la masse lipidique unique en plusieurs inclusions, alors que les inclusions lipidiques multiples ne paraissent pas fusionner ou rarement au cours de l'amaigrissement.

Au fur et à mesure de la diminution de volume de la masse ou des masses lipidiques (fig. 7, Io), l'augmentation de l'osmiophilie, qui sera discutée ultérieurement, est d'autant plus prononcée que la quantité de lipides mobilisés est plus importante et que le rapport quantitatif entre lipides mobilisés et lipides restants est plus grand.

La poursuite de la mobilisation des lipides et la diminution de leur volume se traduisent à un moment donné par l'apparition d'une substance résiduelle, de forme circulaire très fortement osmiophile (fig. II). L'importance quantitative de cette substance au moment de la disparition de ce que l'on peut considérer comme lipides d'accumulation est d'autant plus grande qu'elle résulte d'une quantité plus importante de lipides.

La disparition des inclusions lipidiques pour l'ensemble de la population des cellules adipeuses comme pour chaque cellule est échelonnée dans le temps, ce qui permet parfois d'observer sur une même coupe des inclusions lipidiques de différente importance et la substance résiduelle (fig. I2). Au stade de leur apparition, les résidus lipidiques paraissent contenir une importante quantité de lipofuscine mais leur aspect, et probablement leur composition, comme nous le verrons à l'occasion de l'étude des cellules adipeuses entièrement dépourvues de lipides, sont en continuels changements.

\section{Taille et forme des cellules adipeuses.}

Comme conséquence directe du départ des lipides, la taille des cellules adipeuses décroît jusqu'à disparition totale de ces lipides d'accumulation. Toutefois, la comparaison entre les multiples mesures effectuées sur des cellules ayant une quantité de lipides comparable dans le dépôt normal et au cours de l'amaigrissement, indique que la diminution du volume cellulaire reste toujours inférieure à celle du volume des lipides mobilisés. Ainsi, pour des volumes équivalents de lipides, les cellules adipeuses au cours de l'amaigrissement ont un diamètre toujours supérieur à celui des cellules prélevées sur les animaux à régime normal. De toute évidence, la différence de cas volumes ne correspond pas à un enrichissement en éléments cellulaires pendant le départ des lipides, mais elle traduit la difficulté pour les cellules adipeuses à réđuire leur volume. 
Comme nous l'avons constaté, les cellules adipeuses, pour combler le vide laissé par le départ des lipides, diminuent leur volume par différents processus tels que plissements, aplatissements et contractions, ce qui a pour conséquence le changement continu de la forme cellulaire $L$ 'aspect de la forme cellulaire aux différents stades des transformations dépend, dans la majorité des cas, de la forme de la cellule au moment de la mise au régime des animaux. Néanmoins, mises à part les projections cytoplasmiques, la forme des cellules au cours de cette phase de la mobilisation des lipides est, dans le plus grand nombre de cas, fusiforme, de pourtour souvent irrégulier (fig. 13) ; les formes ovales et ramassées ne sont cependant pas l'exception (fig. II).

\section{Lame basale.}

Le mécanisme de l'évolution de la lame basale des cellules adipeuses au cours de la mobilisation des lipides, donnant lieu à une autre publication, ne sera évoqué ici que brièvement.

Mis à part les adipocytes en état de dégradation, la lame basale devient généralement plus apparente chez toutes les cellules adipeuses dès le début de l'amaigrissement et tend, par la suite, à s'adapter à la régression de la surface cellulaire. Au stade de l'apparition des projections cytoplasmiques, la lame basale est présente sur leur pourtour (fig. $7,8,9$ ), mais elle ne suit pas toujours la rapidité avec laquelle les projections évoluent. Dans certains cas, elle se détache de la membrane plasmique et ses prolongements sont souvent plus importants que les projections cytoplasmiques. Sur la surface cellulaire, en dehors des projections cytoplasmiques et parfois sur leur pourtour, la lame basale fait ses propres plissements (fig I4) dont l'évolution est en rapport avec la régression de la surface de la membrane plasmique. L'importance de l'amplitude de ces plissements, liée à la vitesse de diminution de la surface cellulaire, est parfois supérieure à I $\mu$ (fig. I4). Le rapprochement entre les plissements de la lame basale est généralement rapide, de sorte qu'ils se confondent et forment une enveloppe sur le pourtour cellulaire ayant un aspect peu commun avec la lame basale observée habituellement autour des cellules adipeuses (fig I3, I4). Par la suite, la régression de la surface cellulaire devenant moins rapide, la lame basale, tout en continuant de diminuer sa surface, devient en même temps moins épaisse. Ce changement d'épaisseur de la lame basale, suivant un mécanisme non éluciđé, est dans la plupart des cas irrégulier à un moment donné sur le pourtour d'une même cellule (fig. I3).

\section{Membrane plasmique}

La membrane plasmique des adipocytes et des préadipocytes des dépôts adipeux normaux est souvent pourvue de microinvaginations mais la présence de celle-ci devient générale et infiniment plus abondante pendant la mobilisation des lipides (fig. 9, IO, II, I2, I3, I4) Les microinvaginations de taille variable sont généralement remplies de substance dont le contraste est semblable à celui de la lame basale et parfois supérieur. Les invaginations, simples au début, prennent rapidement la forme de flacons, puis elles se détachent sous forme de vésicules dont la dégradation au début de la déplétion lipidique est très rapide. Ces processus de pinocytose contribuent, de toute évidence, à la diminution des'surfaces cellulaires et à l'enrichissement de la substance cytoplasmique. Comme pour la lame basale et dans la plupart des cas de façon coordonnée, l'évolution de la membrane plasmique est variable 
suivant les cellules. De même, l'évolution de la membrane plasmique est souvent inégale sur le pourtour d'une même cellule en fonction de l'emplacement des éléments extracellulaires et de l'importance des inclusions lipidiques à proximité.

Ainsi, la surface d'une cellule à proximité d'un capillaire ou d'une autre cellule adipeuse a, sur cette partie de la membrane plasmique, bien plus d'invaginations que sur le reste de son pourtour. Il en est de même pour la partie de la membrane plasmique bordant d'importantes inclusions lipidiques.

\section{5. Éléments cytoplasmiques.}

- Le noyau des adipocytes dont l'emplacement, à de rares exceptions, est périphérique, se déplace au cours de la mobilisation des lipides vers l'intérieur de la cellule. Sa forme, généralement en croissant ou triangulaire, prend un aspect plus ou moins ovale et apparemment plus volumineux. Le nucléoplasme uniforme devient plus contrasté et on observe l'apparition d'agglomérats de chromatine principalement disposés sur la périphérie (fig. II, I3, I4). Les nucléoles, au nombre de uri, deux ou trois par noyau, sont de plus en plus apparents (fig. II, I4).

- Les mitochondries, pour la plupart en forme de bâtonnets ou sphériques, sont disséminées de façon irrégulière, de sorte que certaines régions de la cellule adipeuse en sont dépourvues ou presque et qu'elles sont relativement abondantes dans d'autres (fig. II, I3). D'une façon générale, on peut estimer que le nombre de mitochondries par cellule est d'autant moins élevé que la cellule au début de l'amaigrissement était plus évoluée. Les crêtes mitochondriales, orientées en majorité obliquement par rapport au grand axe, sont plus régulières et plus apparentes que chez les adipocytes du régime normal. Toutefois, dans certaines cellules, on observe des mitochondries pratiquement dépourvues de crêtes; ces mitochondries sont généralement sphériques et de taille plus grande (fig. I2).

- L'appareil de Golgi, dans la plupart des cas, dégradé chez les adipocytes au début du régime, est observé dans un nombre important de cellules à partir d'un stade avancé de la déplétion lipidique. Dans la majorité des cellules, un seul ou plus exceptionnellement plusieurs agglomérats de saccules en nombre réduit sont observés à proximité du noyau. Cependant, dans certains cas, cet organite est observé dans les extensions cytoplasmiques.

- Le réticulum endoplasmique rugueux (RER) est très réduit ou inexistant dans la majorité des cellules adipeuses à ce stade d'amaigrissement; il en est de même pour les ribosomes libres. Le réticulum endoplasmique lisse (REL), variable suivant les cellules, est peu abondant. On note la présence d'innombrables microvésicules lisses qui, en raison de leur structure, nous semblent être surtout d'origine pinocytotique.

- La matrice fondamentale du cytoplasme est d'aspect variable d'une cellule à 1'autre, généralement d'autant moins contrastée que la masse lipidique mobilisée a été plus importante.

A côté de ces éléments cytoplasmiques permanents mentionnés, on observe, suivant le degré de déplétion des lipides, un nombre croissant de lysosomes, de corps multivésiculaires, de corps denses et de résidus lipidiques, éléments que l'on retrouvera dans les cellules entièrement dépourvues de lipides.

Au terme de la seconde étape de l'amaigrissement, les cellules adipeuses des animaux ayant perdu environ 50 p. roo de leur poids sont, sauf exception, totale- 
ment dépourvues de lipides, tandis que leur nombre, évalué grossièrement, ne semble pas changer durant cette étape. Les postadipocytes ont leurs propres caractéristiques structurale et ultrastructurale dont l'étude sera poursuivie, ainsi que celle concernant leur fonctionnement après la remise des animaux amaigris à un régime normal.

\section{DISCUSSION}

Les observations effectuées sur des coupes de dépôt adipeux mésentérique de rats aux différents stades de la déplétion lipidique et comparées aux coupes de tissu de rats témoins, nous ont permis de mettre en évidence l'évolution structurale et ultrastructurale des cellules adipeuses à graisse blanche au cours de la mobilisation des lipides d'accumulation qui ne se renouvellent pas. Malgré les difficultés techniques relatives à la préparation des tissus adipeux pour les coupes ultrafines (Vonovar, DeSNOYers, Françors, I97I), et le polymorphisme des cellules avec accumulation lipidique à tous les stades de l'évolution des dépôts (Vodovar, Desnoyers, Flanzy, 1972), nous espérons avoir apporté une image assez fidèle des transformations des populations de cellules adipeuses mésentériques au cours de la déplétion lipidique.

\section{A. - Transformations et devenir des cellules adipeuses au cours de la première étape de la déplétion lipidique}

La brève période d'environ cinq jours, au début du régime de restrictions caloriques, au cours de laquelle les cellules adipeuses gardent le même aspect, est interprétée comme le moment pendant lequel ces cellules sont capables, dans nos conditions d'expérience, d'éviter les transformations apparentes dues au changement de régime. L'intervalle de temps correspondant à la perte d'environ 5 à $20 \mathrm{p}$. Ioo du poids initial des animaux semble être, dans ces conditions d'expérience, la phase la plus critique de la déplétion lipidique. En effet, comme il a été constaté, des perturbations parfois très spectaculaires ont pour conséquence la disparition d'un certain nombre d'adipocytes et le changement brutal et rapide de l'aspect des cellules contenant des inclusions lipidiques en quantité importante. La diversité du degré de maturation des cellules adipeuses au début du régime permet d'expliquer la multiplicité des modes de transformation de ces cellules et de comprendre les raisons de la disparition d'un certain nombre d'adipocytes.

Les travaux, sur la transformation et le devenir des cellules adipeuses au cours de la déplétion lipidique, auxquels nous aurions pu confronter nos investigations, sont absents ; on note cependant que des aspects cellulaires semblables à nos observations pendant la première phase de la mobilisation lipidique, notamment de multiples projections cytoplasmiques, ont été observés sur les cellules des dépôts adipeux épididymaires de rats ayant entièrement perdu leurs lipides d'accumulation (Sheldon, Holitenberg, Winegrad, ig62; Willitamson, I964; Willitamson, LACY, I965). Du fait que ces derniers travaux ont été effectués sur des animaux totalement privés de nourriture, on peut penser que la déplétion lipidique totale a été très rapide et que les transformations des cellules adipeuses n'ont pas pu 
s'effectuer de façon comparable à celles mises en évidence lors de nos investigations faites dans des conditions physiologiques quasi normales. Cela permet de constater que les cellules adipeuses sont toujours soumises à des difficultés d'adaptation, quel que soit le mode de mobilisation des lipides mais que, si le régime amaigrissant est très sévère, les perturbations sont beaucoup plus difficiles à surmonter et sont observées pendant tout le temps de la déplétion lipidique. L'expérimentation avec un régime encore moins restrictif que le nôtre permettrait de voir si on peut parvenir à atténuer les perturbations chez les cellules observées au cours de la première étape. du départ des lipides.

Les travaux effectués sur plusieurs tissus de rats amaigris par un régime pauvre en calories jusqu'à la perte totale des lipides (SimoN, WilliamSoN, I967) montrent que les cellules adipeuses dépourvues de lipides d'accumulation ont une forme généralement allongée et que leur aspect n'est pas comparable à celui décrit à la suite d'un amaigrissement aigu. Cela indique que les transformations ont lieu au cours de la déplétion des lipides ce qui corroboze nos investigations. Les co!lules du dépôt adipeux épididymaire de rats dépourvues de lipides d'accumulation far une restriction calorique (NAPOLITANO, GAGNE, I963) semblent avoir une évolution intermédiaire entre celle des animaux ayant été entièrement privés de nourriture (WIr,IAMSON, I964) et celle des animaux décrits par Simon et WILLIAMSon ce qui laisse à supposer que le régime de NAPOLITANo et GAGNE, non précisé dans leurs travaux, a été plus sévère.

La dégradation et la disparition des adipocytes observés, sauf exception, seulement au cours de la première phase de l'amaigrissement ont été évaluées d'une façon très approximative; néanmoins, nous pensons que leur nombre, dans nos conditions de travail, reste toujours inférieur à Io $\mathrm{p}$. Ioo de la totalité des cellules avec des inclusions lipidiques. Il ressort que go p. Ioo au moins des cellules adipeuses sont capables de se régénérer quand leurs lipides d'accumulation sont mobilisés. Les cellules disparues étant parvenues au stade terminal de leur maturation (Vodovar, DESNOYERS, FlaNZY, I972), nous pensons, en tenant compte des observations des coupes de rats témoins, que leur renouvellement aurait été inéluctable si les animaux n'avaient pas été soumis au régime restrictif. Cela confirme les travaux antérieurs sur le renouvellement cellulaire dans les dépôts adipeux (LEBLOND, MESSIER, KopRIva, I959; Heliman, Heliferström, I96I ; Vodovar, Desnoyers, Flanzy, i972). L'importance quantitative de ces renouvellements, non précisés à ce jour, nous paraît varier, parmi d'autres facteurs, en fonction de l'âge de l'animal, de l'importance $d u$ dépôt et $d u$ régime alimentaire. Le problème de la disparition des adipocytes suivant la sévérité du régime amaigrissant reste à étudier ainsi que l'importance d'éventuelles proliférations des préadipocytes chez les animaux amaigris remis à un régime normal.

Toute appréciation au cours de la première étape de l'amaigrissement concernant l'importance quantitative de la déplétion lipidique des cellules, en fonction des différents stades de maturation, nous paraît délicate et sujette à caution. En effet, pendant cette étape, la taille des cellules, leur masse lipidique et leur distribution topographique sont très différentes. En ce qui concerne l'aspect qualitatif, l'accroissement constant de l'osmiophilie pendant la mobilisation des lipides nous semble confirmer la mobilisation, en priorité, des acides gras saturés. 


\section{B. - Évolution des cellules adipeuses au cours de la seconde étape de la déplétion lipidique}

Une évolution relativement harmonieuse est observée sur la population de cellules contenant des inclusions lipidiques à partir du moment où les animaux ont perdu environ $20 \mathrm{p}$. Ioo de leur poids initial. Cela pourrait être interprété par le fait que les cellules adipeuses, dont les degrés d'évolution sont différents au début du régime, ont perdu un taux de lipides variable et sont parvenus à un stade où le rythme de leur régénération est devenu comparable. Ceci indique qu'à ce moment la dégradation des cellules adipeuses ne se fait plus ou se fait rarement et que la diminution du volume cellulaire en rapport avec le départ des lipides n'occasionne plus de perturbations graves.

Grâce à cette uniformité relative de l'évolution, les transformations structurales et ultrastructurales sont suivies avec moins de difficultés. De ce fait, nous avons pu mettre en évidence le mécanisme de la transformation de la lame basale et expliquer les différents stades de son adaptation au départ des lipides, ce qui n'a pu être fait en étudiant les cellules adipeuses au stade de leur déplétion lipidique totale (NAPOlitano, GaGne ,I963; Williamson, Lacy, I965; Simon, Williamson, I967). Les microinvaginations de la membrane plasmique des cellules adipeuses qui sont particulièrement nombreuses pendant la déplétion lipidique sont surtout interprétées, en accord avec les travaux antérieurs (WASSERMANN, MAC DoNALD, I96o $b$; NAPOIITANO, GAGNE, I963) comme le moyen, pour les cellules, de diminuer leur surface en s'adaptant à la régression du volume consécutif au départ des lipides.

I1 est nécessaire de préciser que les transformations, dont nous avons donné les étapes et qui sont valables dans les conditions de notre travail, ne seront pas obligatoirement identiques si les conditions d'expérience changent, et, en particulier, on peut prévoir leur modification certaine si l'âge de l'animal, l'importance du dépôt et le régime ne sont pas semblables.

Dans le cas des présentes investigations, les animaux ayant perdu environ 50 p. Ioo de leur poids initial ont leurs cellules adipeuses pratiquement dépourvues de lipiđes d'accumulation. Leur aspect ultrastructural, qui sera étudié ultérieurement, ainsi que leurs résidus lipidiques, sont relativement homogènes.

Reçu pour publication en septembre 1972.

\section{SUMMARY}

\section{CHANGES AND FURTHER BEHAVIOUR}

OF THE ADIPOSE CELIS DURING EMACIATION OF RATS

Using $\mathrm{I}_{5}$ weeks old rats weighing about $400 \mathrm{~g}$, the ultrastructural changes of the adipose cells and their further behaviour were studied during lipid depletion caused by a prolonged administration of a caloric restrictive diet and under conditions being as physiological as possible.

It has been shown that the adipocytes, being in the terminal stage of development when the animals were put on the diet, were degraded and generally disappeared before these animals had lost 20 per cent of their initial weight. 
The rest of the adipose cells of the deposits, representing about go per cent of all the cells with lipid inclusions, was subjected to structural and ultrastructural changes allowing their regeneration with simultaneous loss of their lipid accumulations.

It has been noted that the structural and ultrastructural changes of these adipose cells during the loss of their lipids were all the more marked as the lipid inclusions were larger when the animals were put on the diet.

The different stages of these changes until total lipid depletion, generally finished when the animals have lost 50 per cent of their initial weight, are described and discussed.

\section{RÉFÉRENCES BIBLIOGRAPHIQUES}

Hellman B., Hellerström C., r96r. Cell renewal in the white and brown fat tissue of the rat. Acta Path. Microbiol. scand., 51, 347-353.

Leblond C. P., Messier B., Kopriva B., I959. Thymidine- $\mathrm{H}^{3}$ as a tool for the investigation of the renewal of cell populations. Lab. Invest., 8, 296-308.

Millonig G., I96I. The advantages of a phosphate buffer for $\mathrm{OsO}_{4}$ solutions in fixation. J. appl. Physics, 32, I637.

Napolitano L., I963. The differentiation of white adipose cells : an electron microscope study. $J$. Cell. Biol., 18, 663-679.

Napolitano L., Gagne H. T., r963. Lipid depleted white adipose cells : an electron microscope study. Anat. Rec., 147, 273-294.

Sheldon H., Hollenberg C. H., Winegrad A. I., I962. Observations on the morphology of adipose tissue. Diabetes, 11, 378-387.

Simon T. L., Williamson J. R., r967. The fate of lipid-depleted fat cells. Arch. Path., 88, r62-168.

Vodovar N., Desnoyers F., François A. C., I97I. Origine et évolution des adipocytes mésentériques du porcelet avant la naissance. Aspect ultrastructural. $J$. Microscopie, 11, 265-284.

Vodovar N., Desnoyers F., Flanzy J., r972. Dépôt adipeux mésentérique du porcelet. Étude morphologique. Ann. Biol. anim. Bioch. Biophys., 12, 243-262.

Wasserman F., MacDonald T. F., I 960 a. Electron microscopic study of the fine structure of the fat cell as related to function. Argone Nat. Lab., ANL, 6264, 68-82.

Wassermani F., MacDonald T. F., I960 $b$. Electron microscopic investigations of the surface membrane structures of the fat cell and of their changes during dépletion of the cell. $Z$. Zellforsch., 52, 778-80o.

Wilitamson J. R., I964. Adipose tissue. Morphological changes associated with lipid mobilization. J. Cell Biol., 20, 57-74.

Williamson J. R., LAcy P., 1965. In : Handbook of physiology, adipose tissue, 5, 20I-zio. Renold A. E., Cahili G. F., Jr., Am. Phys. Soc. Washington D. C. 


\section{ABREVIATIONS UTILISEES DANS LES PLANCHES}
A Adipocyte
AP Anneau périphérique
B Lame basale
C Capillaire sanguin
D Corps denses
F Fibres collagènes
L Lipides

M Mitochondrie

MP Membrane plasmique

MV Microvésicules

PC Projection cytoplasmique

VP Microinvaginations

Y Matrice fondamentale du cytoplasme 
Les figures $I$ à 5 représentent les transformations des adipocytes pour lesquels le début de la déplétion lipidique est intervenue au stade terminal de leur maturation.

\section{PLANCHE I}

\section{FIG. I}

Vue partielle de deux adipocytes parvenus au stade terminal de leur maturation. Anneau périphérique AP très réduit. Lipides L. $(\times 7000)$.

\section{Frg. 2}

Un des aspects de la dégradation de l'anneau périphérique $A P$ d'un adipocyte parvenu au stade terminal de sa maturation. On ne distingue ni la lame basale, ni la membrane plasmique, les fibres conjonctives $F$ s'entremêlent avec ce qui reste de 1'anneau périphérique AP. Lipides L. ( $\times 35000)$. 

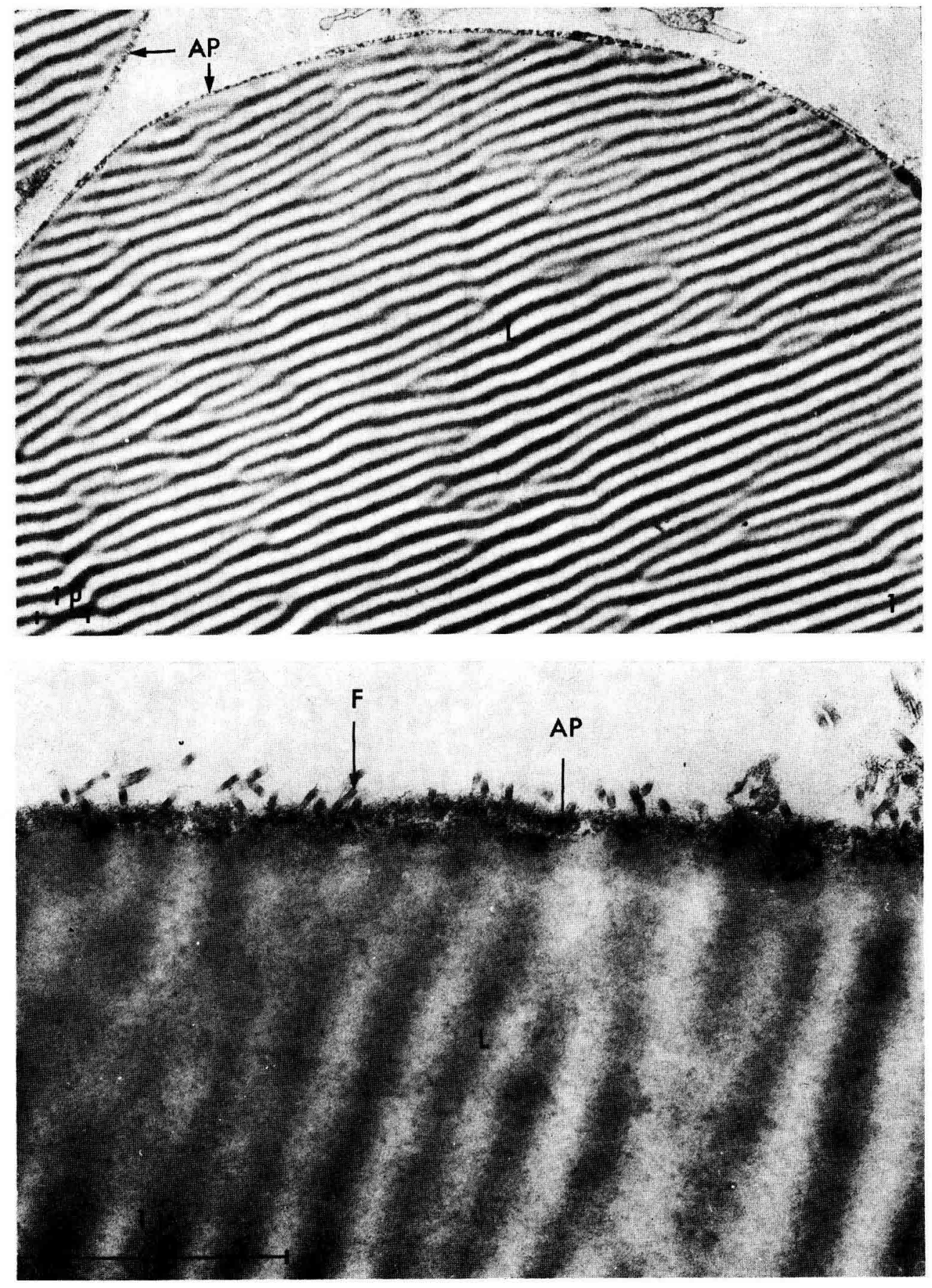


\section{PLANCHE II}

Fig. 3

Vue partielle d'un adipocyte à un stade où la masse lipidique $\mathrm{L}$ est bordée par une enveloppe très fine sans structure apparente. $(\times$ 40.000).

FIG. 4

Vue partielle de la surface d'un adipocyte dont l'anneau périphérique n'est plus apparent. Les fibres conjonctives $F$ viennent directement au contact de la masse lipidique $L$. ( $\times$ 55.500).

\section{FIG. 5}

Vue partielle d'un adipocyte dont la périphérie de la masse lipidique $L$ est presque totalement dépourvue de substance organisée. $(x$ 17.000). 

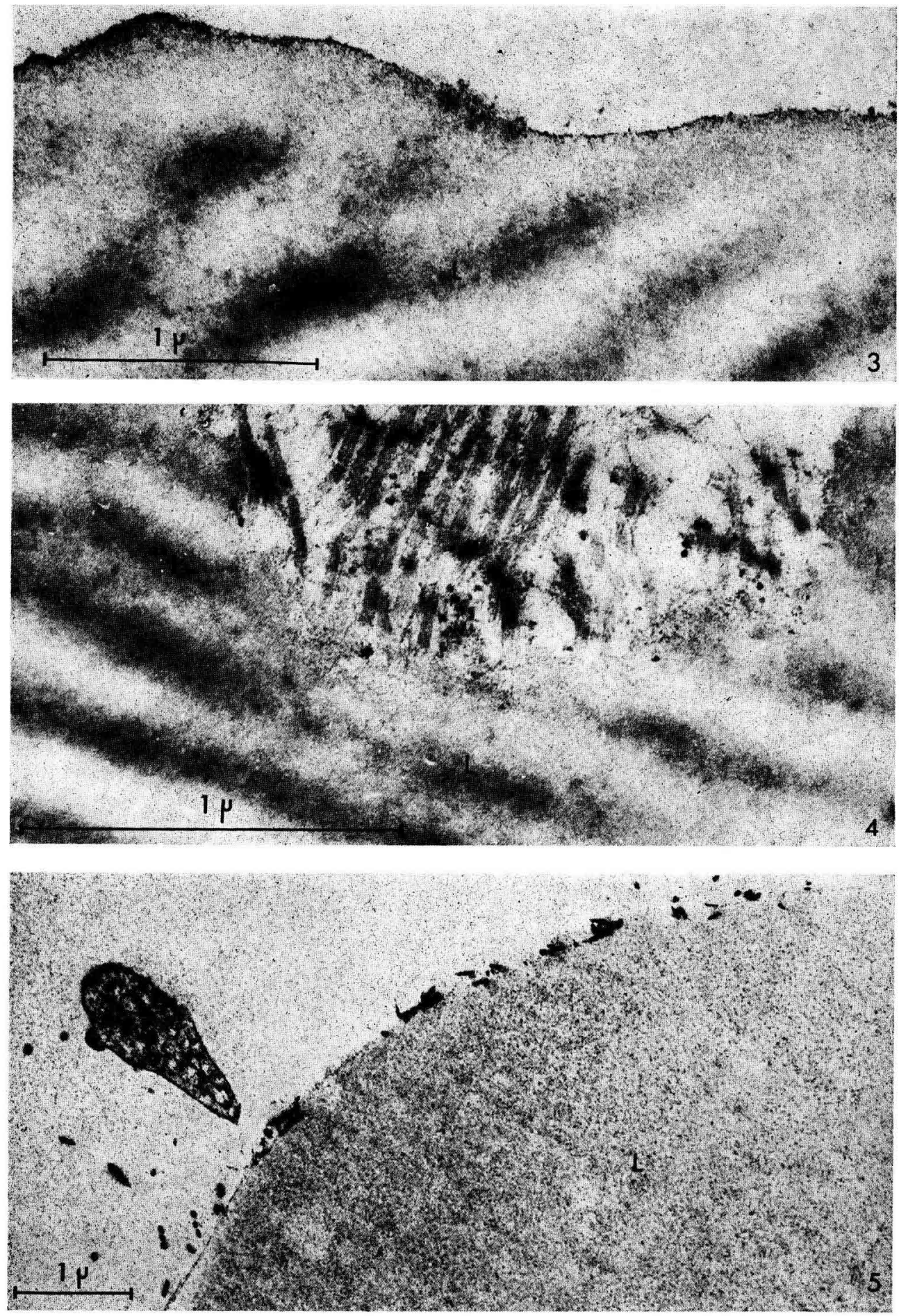

F. DESNOYERS, N. VODOVAR et G. DURAND 


\section{PLANCHE III}

Les figures 6 à 9 représentent les transformations des adipocytes pour lesquels le début de la déplétion lipidique est intervenu au cours de leur maturation.

FIG. 6

Vue partielle de quatre adipocytes entourant un capillaire sanguin $C$. Malgré leur proximité et contrairement au dépôt des animaux à un régime normal, les anneaux périphériques AP sont nettement délimités par leur lame basale $B$, ce qui indique l'individualisation des adipocytes, conséquence de la déplétion lipidique. Lipides : L, mitochondrie : M, Noyau : N. $(\times 8.000)$.

\section{FIG. 7}

Aspect spectaculaire des transformations d'un adipocyte entouré d'autres éléments du dépôt, au cours de la déplétion lipidique. La membrane plasmique MP et la lame basale $B$ sont bien visibles; Les projections cytoplasmiques PC sont de formes variables et l'une d'entre elles montre un regroupement de mitochondries $M$; la matrice fondamentale du cytoplasme $Y$ est de contraste très variable ; la masse lipidique $L$ très osmiophile est bordée d'une zone plus claire. Capillaires sanguins C. $(\times 4.500)$. 

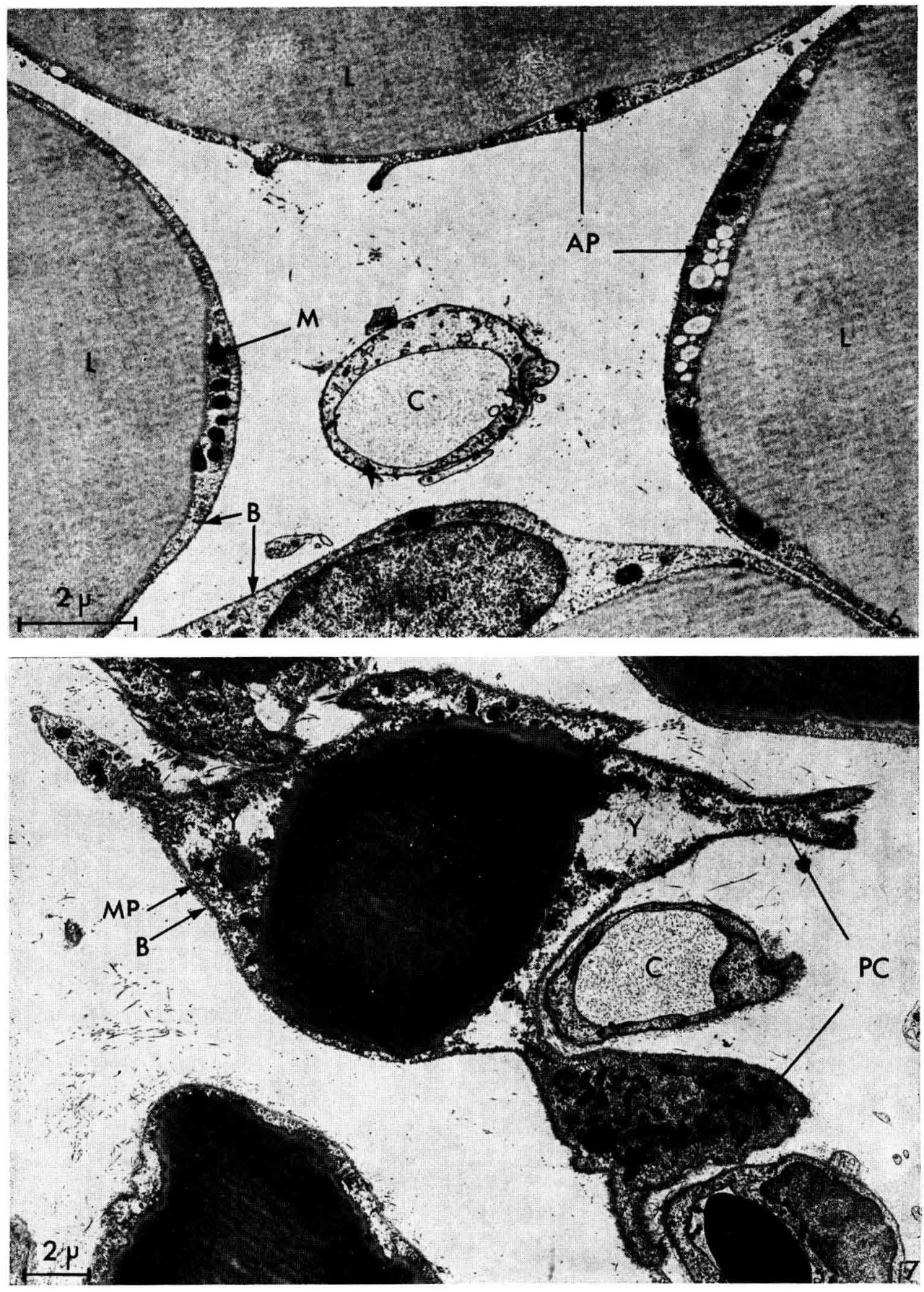


\section{PLANCHE IV}

FIG. 8

Plissements de la surface cellulaire provoqués par la déplétion lipidique d'un adipocyte. Nombreuses microvésicules pinocytotiques MV dans l'anneau périphérique AP. La disparition des lipides $\mathrm{L}$ fera apparaitre des projections cytoplasmiques comparables à celles de la fig. 9 . ( × 18.000).

Fig. 9

Coupe d'une projection cytoplasmique PC d'un adipocyte au cours de la déplétion lipidique. Les microvésicules pinocytotiques MV et les micro-invaginations VP de la membrane plasmique MP sont nombreuses; la lame basale $B$ est bien visible. Masse lipidique $\mathrm{L}$. ( $\times 26.000)$. 


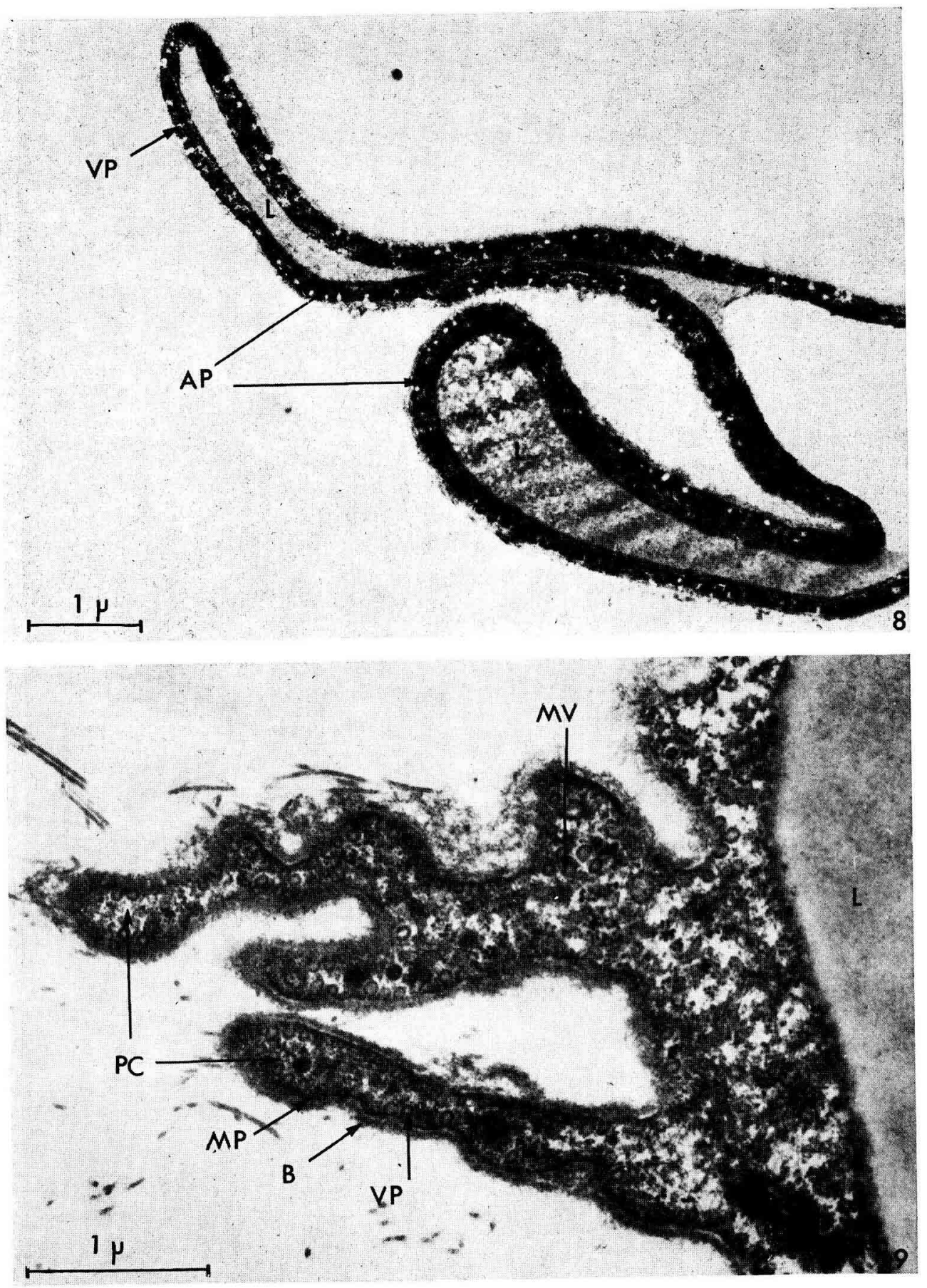




\section{PLANCHE V}

FIG. 10

Coupe d'un préadipocyte au cours de la déplétion lipidique; ses multiples inclusions lipidiques $L$, dont l'osmiophilie a tendance à augmenter sauf en bordure, étaient présentes au début du départ des lipides. $(\times 6.000)$. 


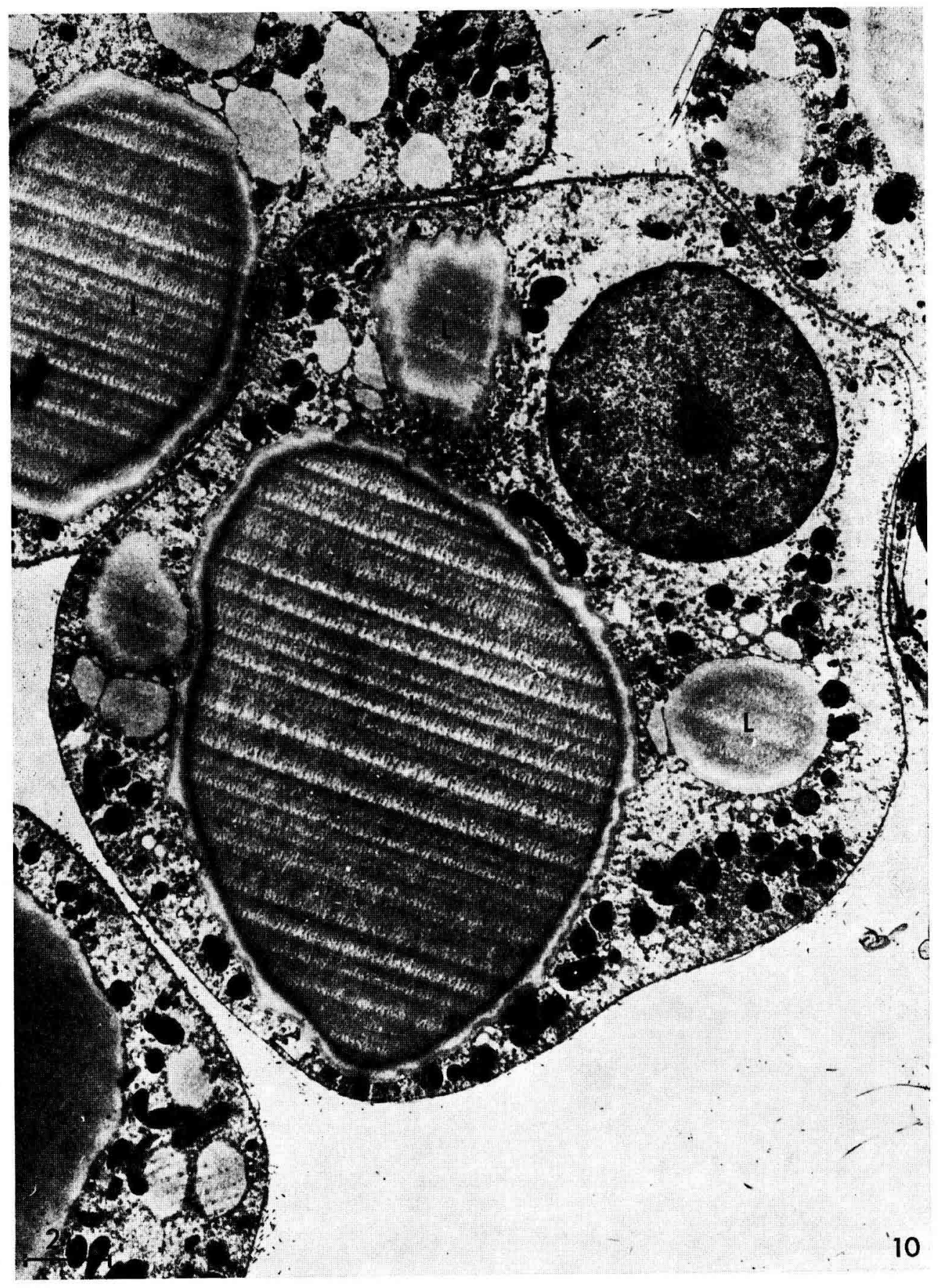




\section{PLANCHE VI}

FIG. 11

Coupe d'un postadipocyte (cellule adipeuse ayant perdu ses lipides d'accumulation) de forme ramassée ; les trois corps denses D sont les résidus de la déplétion lipidique. La lame basale $B$ et la membrane plasmique MP, avec de nombreuses microinvaginations VP, sont bien visibles; la matrice fondamentale du cytoplasme $\mathrm{Y}$ est peu contrastée ; les mitochondries $M$ sont en nombre réduit. Noyau N. ( $\times 9.200)$.

FIG. 12

Coupe d'une cellule adipeuse montrant simultanément des inclusions lipidiques $\mathrm{L}$ et des résidus de la déplétion lipidique $\mathrm{D}$. Les mitochondries $M$ sont d'aspect et de taille variables. $(\times 22.000)$. 


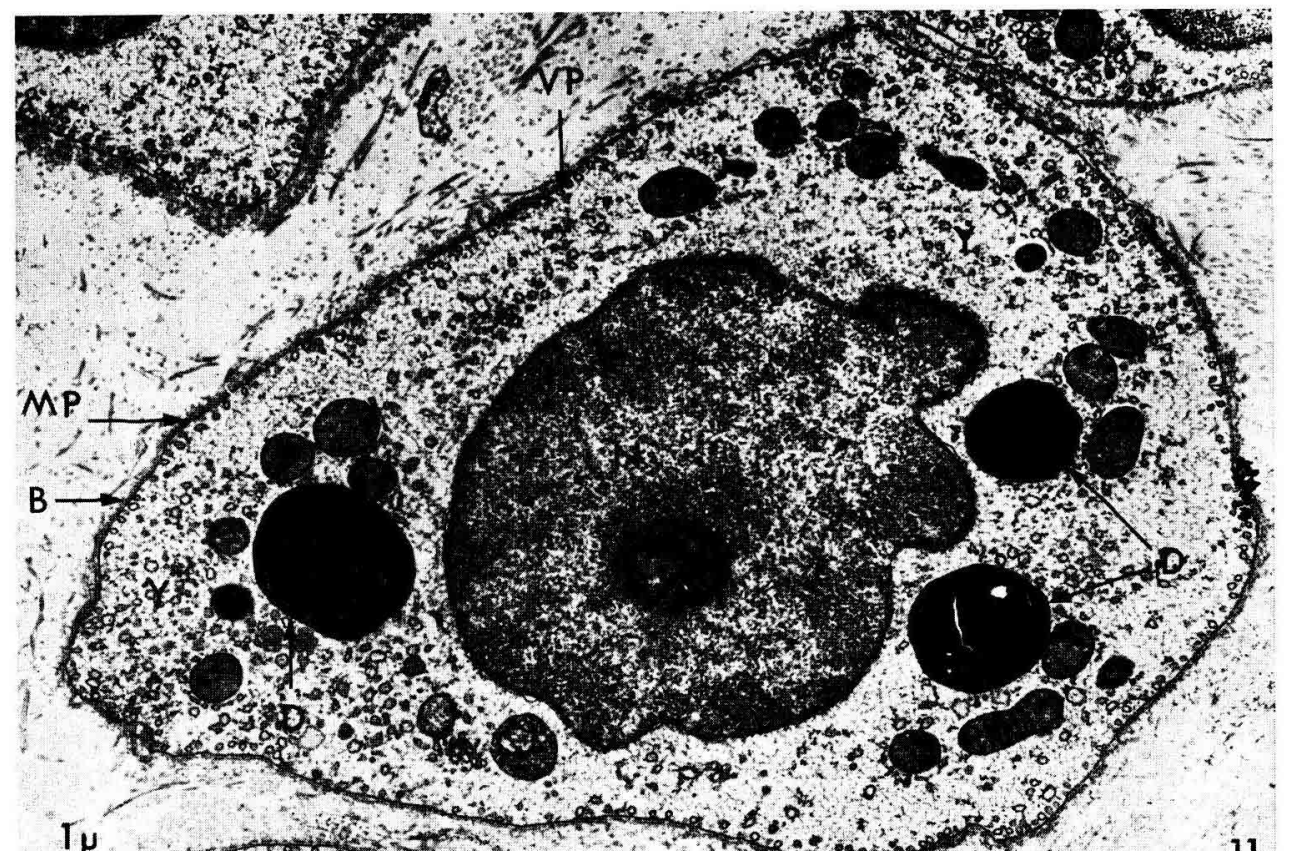
$r_{p}+4$

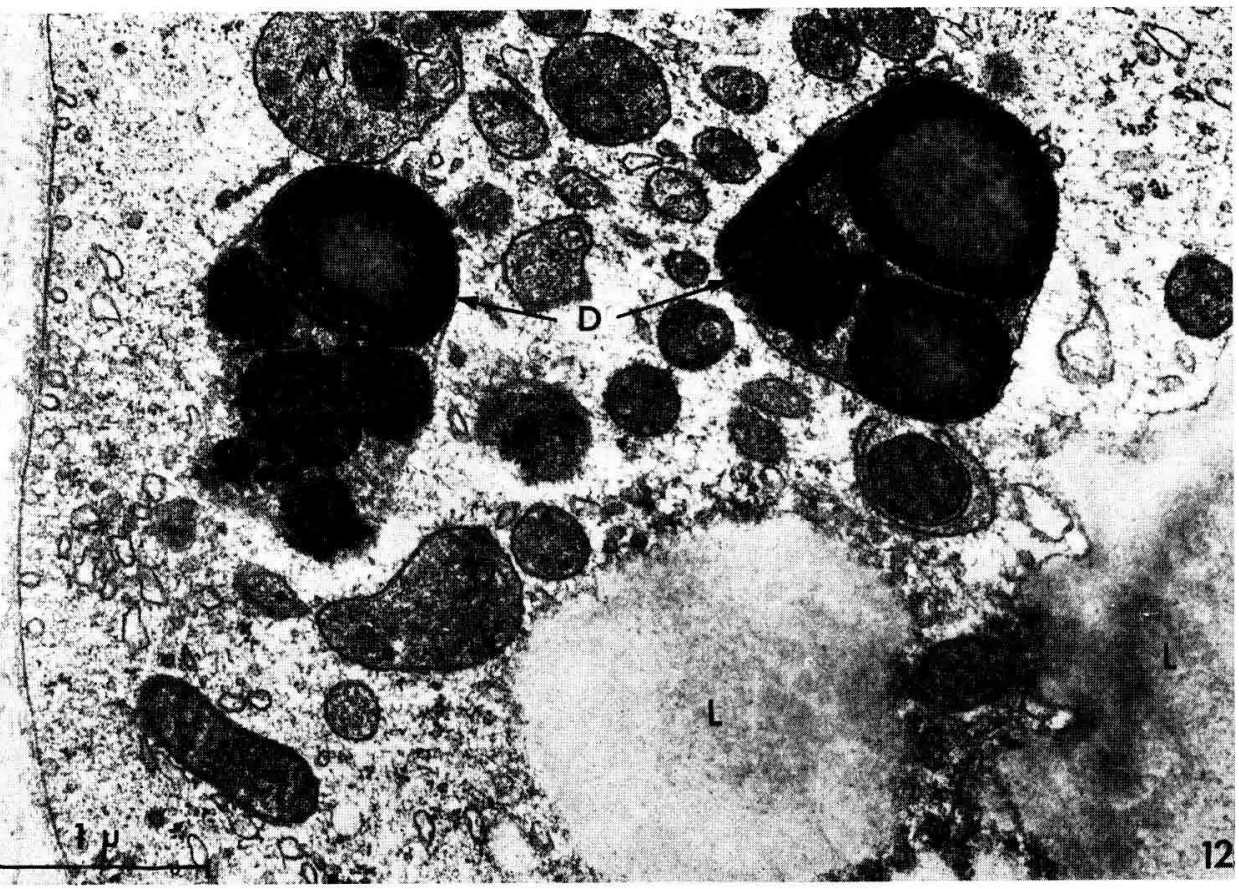




\title{
PLANCHE VII
}

\author{
Fig. 13
}

Vue d'un adipocyte A d'aspect fusiforme à un stade avancé de la déplétion lipidique. Lipides $L$; Noyau $N$; mitochondries $M$; membrane plasmique MP ; lame basale $B .(\times 7.200)$.

FIG. 14

Adipocyte dans lequel il ne reste que deux petites inclusions lipidiques $L$. Les innombrables plissements de la lame basale B indiquent dans quelles proportions la cellule a diminué de volume. Noyau N ; mitochondrie M. ( $\times 3.000)$. 

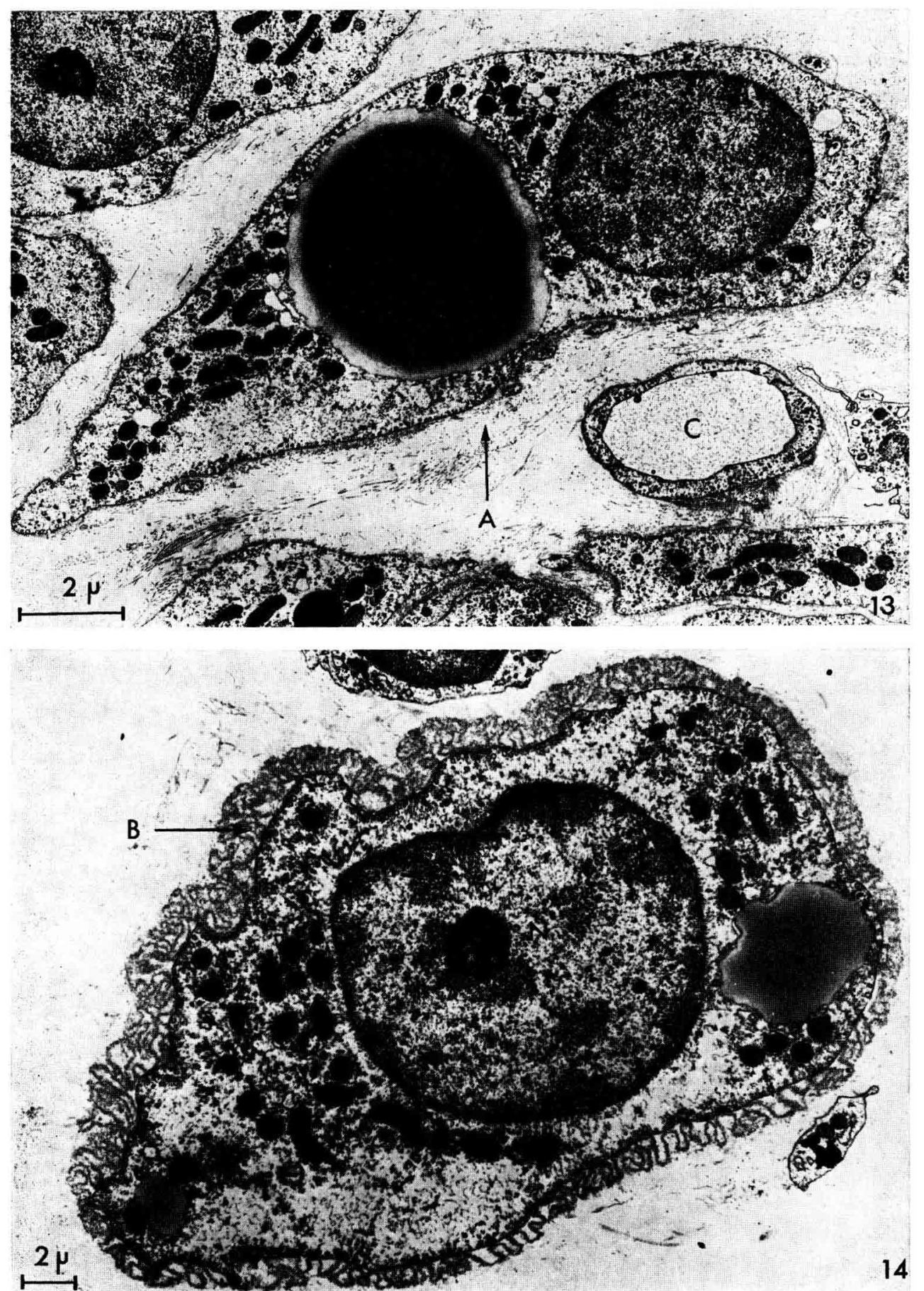